\title{
Volumetric and Functional Heterogeneity of Human Monocytes
}

\author{
Edward B. Arenson, Jr., Martin B. Epstein, and Robert C. Seeger, Department of \\ Pediatrics and Department of Radiological Sciences, University of California, \\ Los Angeles School of Medicine and Physics Department, California State \\ University, Los Angeles, Los Angeles, California 90024
}

A B S T RACT Volume analysis of purified human blood monocytes revealed distinct populations of large and small cells. Computer curve fitting suggested a third, intermediate-sized population. These monocytes were designated $\mathbf{M}_{1}, \mathrm{M}_{2}$, and $\mathrm{M}_{3}$ in order of increasing size, and their approximate volumes were 150,250 , and $480 \mu \mathrm{m}^{3}$, respectively. The three subpopulations were present in all 30 normal individuals tested. Two new techniques were developed that separate monocytes into $M_{1}+M_{2}$ and $M_{3}$ fractions; one used preferential incorporation of carbonyl iron particles by $\mathrm{M}_{3}$ cells and the other used the selective aggregation of $\mathrm{M}_{3}$ cells by thrombin in the presence of platelets. The chemotactic response to zymosan-activated human serum by total monocytes, $\mathrm{M}_{1}+\mathrm{M}_{2}$ monocytes, and $\mathrm{M}_{3}$ monocytes was determined by the agarose plate method. In all experiments $\mathbf{M}_{3}$ monocytes were 10 fold more responsive than $\mathbf{M}_{1}+\mathbf{M}_{2}$ monocytes and were significantly more so than total monocytes. These findings suggest that $\mathbf{M}_{3}$ cells are the major subpopulation capable of directional migration. This investigation establishes the existence of volumetrically definable subpopulations of human monocytes that are functionally distinct.

This work was presented in part at the 63rd Annual Meeting of the Federation of American Societies of Experimental Biology, Dallas Texas, April 1979 and published as an abstract in 1979. Fed. Proc. 38: 1170.

Dr. Arenson was initially supported by National Institutes of Health training grant $\mathrm{Ca} 09120$ and is currently supported by CA23175 and CA16042 from the Natinal Cancer Institute, Department of Health, Education, and Welfare. Dr. Epstein was partially supported by Associated Western Universities and Dr. Seeger is the recipient of Research Career Development award CA00069 from the National Cancer Institute, Department of Health, Education, and Welfare. Address requests for reprints to Dr. Arenson.

Received for publication 26 June 1979 and in revised form 19 November 1.97 .9 .

\section{INTRODUCTION}

The mononuclear phagocyte system includes bone marrow promonocytes, circulating blood monocytes, and tissue macrophages. The spectrum of functions attributable to mononuclear phagocytes has broadened markedly, and there is increasing evidence that these cells are metabolically, physically, and functionally heterogeneous (1). In this context, it is reasonable to hypothesize that the mononuclear phagocyte system contains unrecognized subpopulations of cells that are functionally distinct. Experiments reported in this communication demonstrate that human peripheral blood contains volumetrically distinct subpopulations of monocytes with markedly different chemotactic capabilities.

\section{METHODS}

Monocyte purification. Washed mononuclear leukocytes $(\mathrm{MNL})^{1}$ that contained lymphocytes and monocytes obtained by centrifugation of heparinized blood over Ficoll-Hypaque (Pharmacia Fine Chemicals, Inc., Piscataway, N. J.) (2) were suspended in RPMI-1640 medium (Grand Island Biological Co., Grand Island, N. Y.) that contained 20\% human AB serum at a concentration of $3-5 \times 10^{6}$ cells $/ \mathrm{ml} .5 \mathrm{ml}$ of this suspension was placed in flasks (No. 3014 Falcon Labware, Div. of Becton, Dickinson \& Co., Oxnard, Calif.), gassed with $5 \% \mathrm{CO}_{2}$ in air, and centrifuged $\left(5 \mathrm{~min}\right.$ at $60 \mathrm{~g}$ ) onto the $25-\mathrm{cm}^{2}$ plastic surface. After incubation for $30 \mathrm{~min}$ at $37^{\circ} \mathrm{C}$, nonadherent cells were removed by vigorously washing (four times) with warm Hanks' balanced salt solution (HBSS). Adherent cells then were removed by incubation for 10-20 min in RPMI-1640 medium that contained $20 \%$ fetal calf serum (FCS) and $0.4 \%$ lidocaine (Elkins-Sinn, Inc., Cherry Hill, N. J.) followed by gentle squirting. Cells were washed once, counted, and resuspended in HBSS for analysis. Cell populations obtained in this manner were $>90 \%$ viable and con-

\footnotetext{
${ }^{1}$ Abbreviations used in this paper: FCS, fetal calf serum; HBSS, Hanks' balanced salt solution; MNL, mononuclear leukocytes.
} 
tained $89 \pm 5 \%$ monocytes by nonspecific esterase staining (3). Phagocytosis of opsonized yeast or staphylococci was used as an additional monocyte marker in initial experiments and correlated closely with nonspecific esterase staining. Fewer than 5\% granulocytes were present by morphologic examination (Wright's stain), and B cells never exceeded 6\% as determined by fluorescent staining for membrane immunoglobulin. This method isolated virtually all monocytes that were present in MNL, because $<2 \%$ of nonadherent cells were monocytes by nonspecific esterase staining, Wright's stain morphology, and readherence to plastic.

Monocyte volume analysis. Volume analyses were performed on purified monocytes with a multiparameter cell sorter/analyzer built according to the design of Steincamp et al. (4). Before analysis, monocytes were gently flushed through 25-gauge needles and observed microscopically to insure that only single cells were analyzed. Dilute suspensions of monocytes $\left(5 \times 10^{5} / \mathrm{ml}\right.$ in $\left.2 \mathrm{ml}\right)$ were incubated for $10 \mathrm{~min}$ in fluorescein diacetate $(12 \mu \mathrm{g} / \mathrm{ml}$ in HBSS ) and filtered through a $40-\mu \mathrm{m}$ nylon mesh before analysis. Volume analyses were gated on fluorescence to insure that only viable nucleated cells were analyzed (5). Cell numbers were recorded over 200 cell volume channels. Each volume analysis was calibrated with $10-\mu \mathrm{m}$ diameter latex beads (10- $\mu \mathrm{m}$ standard bright fluorespheres; Coulter Electronics, Inc., Hialeah, Fla.

Separation of monocyte subpopulations. Two new methods were developed for separating monocyte subpopulations. The first was based upon the hypothesis that volumetrically defined monocyte subpopulations would differ in their ability to incorporate carbonyl iron particles. Total mononuclear cells were suspended in $5 \mathrm{ml}$ of RPMI-1640 at a concentration of $10 \times 10^{6} / \mathrm{ml}$ and combined with $10 \mathrm{ml}$ of RPMI-1640 that contained $25 \%$ FCS and $0.5 \%$ carbonyl iron (iron powder SF special 11-63771, lot 64; GAF Corp., New York) (6). After incubating on a rotor at $37^{\circ} \mathrm{C}$ for $15 \mathrm{~min}$, iron-laden cells and excess iron were completely removed by passing the suspension over a magnet followed by centrifugation over Ficoll-Hypaque (specific gravity 1.077) at $200 \mathrm{~g}$ for $15 \mathrm{~min}$. Iron-free MNL containing residual monocytes were harvested, and iron-laden cells were discarded. Monocytes in this iron-free MNL preparation were either assayed directly for chemotactic activity or were analyzed volumetrically after purification by adherence. Purified adherent cells were $76 \pm 10 \%$ (10 donors) nonspecific esterase positive.

The second method was based upon the observation that aggregates that contained monocytes formed when MNL were incubated with thrombin in the presence of platelets (7). MNL, prepared from heparinized blood by centrifugation over Ficoll-Hypaque, were suspended in HBSS that contained $0.5 \% \mathrm{FCS}$ at a concentration of $10-20 \times 10^{6} \mathrm{cells} / \mathrm{ml}$ and $1 \mathrm{ml}$ was distributed into polystyrene tubes (No. 4-978-148; Fisher Scientific Co., Pittsburg, Pa.). $10 \mu \mathrm{l}$ of thrombin (100 U/ml in normal saline and 50\% glycerol; ThrombinTopical, lot. 916923A; Parke, Davis \& Co., Detroit, Mich.) was added to each tube. The tubes were capped and rotated at room temperature for 1-2 min until large aggregates of platelets and leukocytes formed. These aggregates formed a pellet after centrifugation $(1,000 \mathrm{~g}, 4 \mathrm{~s}$, Fisher model 59 centrifuge). Cells in the supernatant fluid were placed in clean Fisher tubes, cell pellets were disaggregated and resuspended by gentle pipetting, and both fractions were washed twice with HBSS. Monocytes in pellet and supernatant fractions were assayed directly for chemotactic activity or were analyzed volumetrically after purification by adherence. Purified adherent cells from the supernatant and pellet fractions were $69 \pm 19 \%$ and $93 \pm 2 \%$ nonspecific esterease positive (five donors), respectively.
Monocyte chemotaxis. The chemotactic activity of whole MNL was compared with that of MNL that contained selected monocyte subpopulations. MNL were tested instead of purified monocytes to minimize manipulations that might differentially affect monocyte subpopulations. With the exception of the iron depletion method, in which whole MNL were not exposed to iron, all cell populations were subjected to exactly the same experimental manipulations. Volume analyses were performed on monocytes purified by adherence from each MNL preparation to corroborate successful enrichment for monocyte subpopulations.

Monocyte chemotaxis was assayed by the agarose method as previously reported (8). Briefly, 35-mm petri dishes that contained $0.75 \%$ electrophoresis-grade agarose (Fisher Scientific Co.) in medium 199 with $10 \%$ FCS were prepared, and three wells (4-mm Diam) were punched $2 \mathrm{~mm}$ apart. Unfractionated MNL or MNL enriched for monocyte subpopulations by iron depletion or thrombin aggregation were suspended in medium 199 with $10 \%$ FCS and the proportion of monocytes in each preparation was assessed by morphology (Wright's stain) and nonspecific esterase staining. The percentage of monocytes in each preparation was equalized by adding autologous iron-depleted nonadherent lymphocytes (>98\% lymphocytes by Wright's and nonspecific esterase staining), and $10^{5}$ monocytes in $10 \mu \mathrm{l}$ were added to central wells of triplicate plates. $10 \mu$ l of zymosan-activated human serum was added to the left well as the chemoattractant, and $10 \mu \mathrm{l}$ of normal saline was added to the right well as the control. After incubating plates for $16 \mathrm{~h}$ at $37^{\circ} \mathrm{C}$ in an humidified environment that contained $5 \% \mathrm{CO}_{2}$ in air, the number of cells migrating toward the chemoattractant and the control was determined by using a $5 \times 5$-mm microscope eyepiece grid to count cells between central and lateral wells. The mean \pm 1 SD of specifically migrating cells (number toward chemoattractant minus number toward control) was calculated for triplicate plates.

\section{RESULTS}

Identification of monocyte subpopulations. Representative cell volume distributions of purified monocytes from four healthy adults are shown in Fig. 1. Two major peaks of large and small cells were readily apparent. However, computer analysis with optimal Gaussian curve fitting required a third peak between the two major peaks. Hence, three peaks, designated $M_{1}, M_{2}$, and $M_{3}$ in order of increasing size, were used for distribution analysis; their approximate mean volumes were 150,250 , and $480 \mu \mathrm{m}^{3}$, respectively. The volume of $M_{1}$ and to a lesser extent $M_{2}$ monocytes overlapped that of purified lymphocytes, whereas $\mathbf{M}_{3}$ monocytes were significantly larger. A distinct peak of cells of $\mathrm{M}_{3}$ volume was readily identified in whole $\mathrm{MNL}$ preparations. For the purpose of functional analysis, $M_{1}$ and $M_{2}$ monocytes were regarded as a single population, $\mathrm{M}_{1}+\mathrm{M}_{2}$.

A summary of our analysis of purified monocytes from 30 normal individuals is provided in Table $I$. $M_{1}, M_{2}$, and $M_{3}$ cells always were present with percent distributions of $28 \pm 15,23 \pm 14$, and $49 \pm 12$, respectively. The percentage of cells in each of these subpopulations exceeded that of nonmonocytes $(11 \pm 5 \%)$ 


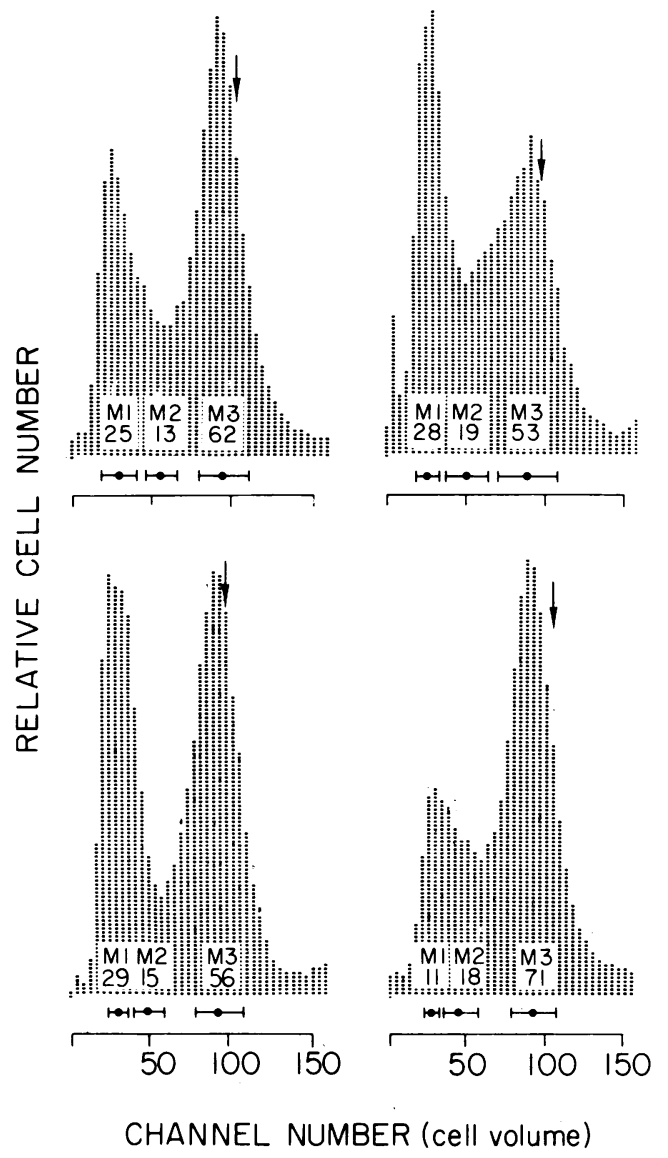

Figure 1 Cell volume distributions of purified monocytes from four normal adults. Channel numbers correlate directly with cell volume. Each distribution was calibrated with $10 \mu \mathrm{m}$ Diam $\left(524 \mu \mathrm{m}^{3}\right)$ latex beads the position of which is indicated by an arrow $(\downarrow)$. Approximate diameters and volumes for $M_{1}, M_{2}$, and $M_{3}$ cells are $7 \mu \mathrm{m}$ and $150 \mu \mathrm{m}^{3}, 8 \mu \mathrm{m}$ and 250 $\mu \mathrm{m}^{3}$, and $9 \mu \mathrm{m}$ and $480 \mu \mathrm{m}^{3}$, respectively. Mean channel No. \pm 1 SD $(\longmapsto-1)$ as well as relative percentage of cells are shown for each peak. To calculate percentages of cells in each peak, volume data were represented by three rather than two Gaussian distributions because three always gave the best fit by chi-square analysis; distribution parameters were optimized with a PDP 11/10 minicomputer using a nonlinear least-squares algorithm; areas under volume peaks were computed using these parameters.

in the adherent cell preparations; therefore, monocytes were abundant in each peak. For individuals of age 20-50 yr, there were no sex-related differences in monocyte volume distributions. Our data for a few healthy individuals of different age groups (Table I) suggested that their monocyte volume distributions were similar to the 20-50-yr group.

To determine if these subpopulations of monocytes were reproducibly present, we performed multiple analyses on several normal individuals. Monocyte subpopulations were consistently present for periods
TABLE I

Percentage of Monocytes in $M_{1}, M_{2}, M_{1}+M_{2}$, and $M_{3}$ Peaks*

\begin{tabular}{lcccc}
\hline & \multicolumn{5}{c}{ Monocytes in each peak } \\
\cline { 2 - 6 } \multicolumn{1}{c}{ Age } & $M_{1}$ & $M_{2}$ & $M_{1}+M_{2}$ & $M_{3}$ \\
\hline \multicolumn{1}{c}{${ }^{\prime}$} & & & & \\
$20-50$ Male $(n=19)$ & $25 \pm 16$ & $25 \pm 14$ & $50 \pm 16$ & $50 \pm 15$ \\
$20-50$ Female $(n=11)$ & $26 \pm 16$ & $21 \pm 15$ & $47 \pm 18$ & $53 \pm 19$ \\
$<10(n=3)$ & $40 \pm 21$ & $18 \pm 12$ & $58 \pm 13$ & $42 \pm 13$ \\
$>50(n=5)$ & $31 \pm 14$ & $16 \pm 17$ & $47 \pm 4$ & $53 \pm 3$ \\
All ages $(n=38)$ & $28 \pm 15$ & $23 \pm 14$ & $51 \pm 15$ & $49 \pm 12$ \\
\end{tabular}

* 30 determinations on 22 individuals between 20 and $50 \mathrm{yr}$ of age were analyzed according to sex. Three determinations on children $<10 \mathrm{yr}$ and five determinations on adults over $50 \mathrm{yr}$ are shown separately for comparison. Each value is mean $\pm 1 \mathrm{SD}$.

up to $6 \mathrm{mo}$; for example, four determinations on one individual resulted in $20 \pm 8,26 \pm 12$, and $54 \pm 10 \% \mathrm{M}_{1}$, $\mathrm{M}_{2}$, and $\mathrm{M}_{3}$ cells, respectively. These experiments also demonstrated that monocyte subpopulations of some individuals could be consistently at one extreme of the normal range (e.g., $\mathbf{M}_{1}, \mathbf{M}_{2}$, and $\mathbf{M}_{3}$ of $17 \pm 3$, $4 \pm 5$, and $79 \pm 8 \%$ ).

Separation of monocyte subpopulations. Methods were developed for separating $M_{1}+M_{2}$ and $M_{3}$ subpopulations so that functional capabilities could be determined. Incubation of MNL with carbonyl iron particles $\left(15 \mathrm{~min}, 37^{\circ} \mathrm{C}\right)$ and then complete separation of iron-laden cells removed $80 \%$ of the adherent monocytes. Remaining adherent cells were $76 \pm 10 \%$ nonspecific esterase positive, and $\geq 74 \%$ were $M_{1}$ cells by volumetric analysis (Fig. 2). Variable numbers of $\mathrm{M}_{2}$ cells were present, but $\mathrm{M}_{3}$ cells were virtually absent. Incorporation of carbonyl iron by $\mathrm{M}_{3}$ monocytes could be abrogated by performing the incubation at $4^{\circ} \mathrm{C}$ or in the presence of $1 \mathrm{mM} \mathrm{N}$-ethylmaleimide, which prevents phagocytosis (9). This suggested that the differential ability of monocyte subpopulations to incorporate carbonyl iron involved phagocytic diversity rather than a simple difference in surface area and binding properties.

A second method was developed with thrombin, which provided $M_{1}+M_{2}$ and $M_{3}$ subpopulations. Incubation of MNL with thrombin in the presence of platelets caused the formation of aggregates that contained monocytes that were readily pelleted by centrifugation (7). Volume analysis of adherent monocytes from both the pellets and supernates demonstrated that the supernatant cells $(69 \pm 19 \%$ nonspecific esterase positive) contained predominantly $\mathrm{M}_{1}+\mathrm{M}_{2}(\geq 80 \%)$ monocytes, whereas those in the pellet $(93 \pm 2 \%$ nonspecific esterase positive) were $\mathrm{M}_{3}$ monocytes ( $\geq 90 \%$ ) (Fig. 3). 


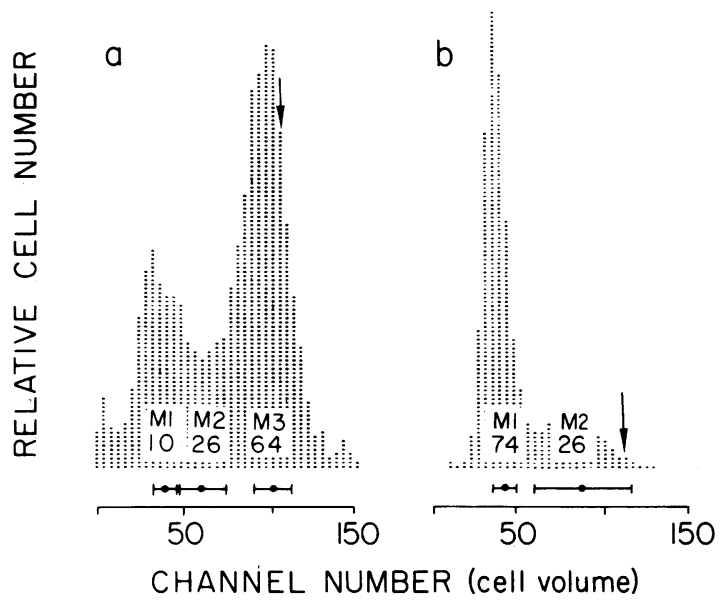

FIGURE 2 Differential incorporation of carbonyl iron particles by monocyte subpopulations. Monocytes were purified from total mononuclear leukocytes $(a)$ and from aliquots of these cells which were previously depleted of iron incorporating cells $(b)$. Iron incorporating cells were removed after incubating $\mathrm{MNL}$ at $37^{\circ} \mathrm{C}$ with $0.33 \%$ iron for $15 \mathrm{~min}$ (b). Volume analysis was performed as detailed in the text and Fig. 1.

Chemotactic responses of monocyte subpopulations. Directional migration is an important function of mononuclear phagocytes. Therefore, we asked whether volumetrically defined subpopulations of monocytes differed in their chemotactic capabilities. To minimize the likelihood that chemotactic differences between monocyte subpopulations were due to purification procedures, two independent methods were used to prepare monocyte subpopulations.

Preferential depletion of $\mathbf{M}_{3}$ monocytes with carbonyl iron allowed the comparison of total monocytes to enriched $\mathrm{M}_{1}+\mathrm{M}_{2}$ monocytes (Table II). The chemotactic response of total monocytes was significantly greater than $M_{1}+M_{2}$ monocytes in all cases. By using the thrombin aggregation technique, total monocytes, enriched $\mathrm{M}_{3}$ monocytes, and enriched $\mathrm{M}_{1}+\mathrm{M}_{2}$ monocytes could be compared (Table III). Brief exposure to thrombin did not significantly alter monocyte chemotaxis because the chemotactic response of untreated total monocytes was the same as thrombin-treated total monocytes. Comparison of monocyte subpopulations demonstrated that the chemotactic response of $\mathrm{M}_{3}$ cells was significantly $(P<0.05)$ greater than total monocytes and 10-fold greater than $\mathrm{M}_{1}+\mathrm{M}_{2}$ cells $(P<0.001)$. This observation corroborated the suggestion from the iron depletion experiments that $\mathrm{M}_{3}$ monocytes were the most chemotactically active subpopulation.

\section{DISCUSSION}

Our experiments clearly demonstrated the existence of large and small human blood monocytes. Because

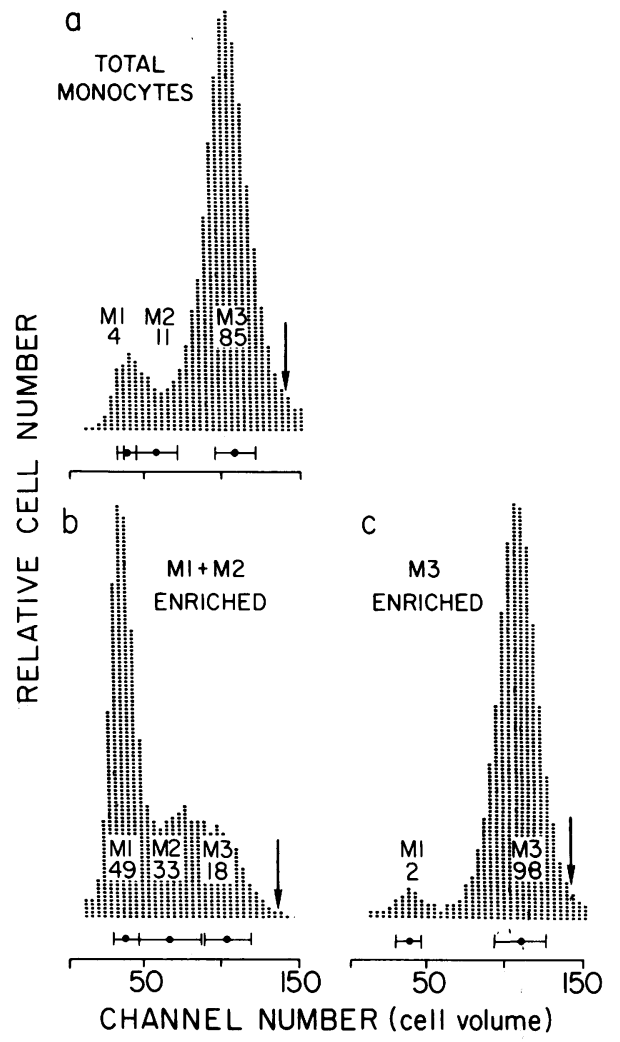

FIGURE 3 Differential aggregation of monocyte subpopulations by thrombin. Monocytes were purified from the pellets and supernates of MNL suspensions after thrombin aggregation and centrifugation as described in the text. $a$ shows the volume distribution of total monocytes after thrombin treatment; although the $M_{1}$ peak appears larger than the $M_{2}$ peak, the $\mathbf{M}_{2}$ peak is actually larger owing to its greater width ( -1 , mean channel No. \pm 1 SD). Volume distributions of supernatant $(b)$ and pellet $(c)$ monocytes demonstrate enrichment of $M_{1}+M_{2}$ monocytes in the supernate and enrichment of $\mathrm{M}_{3}$ monocytes in the pellet.

TABLE II

Chemotactic Activity of Total Monocytes vs. Enriched $M_{1}+M_{2}$ Monocytes Prepared by Iron Depletion Method*

\begin{tabular}{clc}
\hline Donor & \multicolumn{1}{c}{ Cell preparation } & No cells migrating \\
\hline \multirow{2}{*}{1} & Total monocytes & $129 \pm 9$ \\
& $M_{1}+M_{2}$ monocytes & $23 \pm 5$ \\
2 & Total monocytes & $158 \pm 23$ \\
& $M_{1}+M_{2}$ monocytes & $38 \pm 7$ \\
\multirow{2}{*}{3} & Total monocytes & $88 \pm 15$ \\
& $M_{1}+M_{2}$ monocytes & $27 \pm 9$
\end{tabular}

* Mononuclear leukocytes containing either total monocytes or $>72 \% \mathrm{M}_{1}+\mathrm{M}_{2}$ monocytes were compared for their chemotactic activity. $\mathrm{M}_{3}$ monocytes were removed by the iron depletion method.

$\ddagger$ Mean \pm 1 SD of cells migrating specifically toward chemoattractant for triplicate plates. 
TABLE III

Chemotactic Activity of Total, Enriched $M_{3}$, and Enriched $M_{1}+M_{2}$ Monocytes Prepared by Thrombin

Aggregation Method*

\begin{tabular}{clc}
\hline Donor & \multicolumn{1}{c}{ Cell preparation } & Cells migrating \\
\hline 1 & Total monocytes & $104 \pm 6$ \\
& Total monocytes (thrombin) & $109 \pm 13$ \\
& $\mathbf{M}_{3}$ monocytes & $247 \pm 11$ \\
& $\mathbf{M}_{1}+\mathbf{M}_{2}$ monocytes & $22 \pm 5$ \\
2 & Total monocytes & $275 \pm 7$ \\
& $\mathbf{M}_{3}$ monocytes & $306 \pm 17$ \\
& $\mathbf{M}_{1}+\mathbf{M}_{2}$ monocytes & $28 \pm 2$ \\
3 & $T_{0}$ motal mocytes & $134 \pm 9$ \\
& $\mathbf{M}_{3}$ monocytes & $202 \pm 11$ \\
& $\mathbf{M}_{1}+\mathbf{M}_{2}$ monocytes & $17 \pm 3$ \\
\hline
\end{tabular}

* MNL that contained either total monocytes, $\geq 90 \% \quad \mathrm{M}_{3}$ monocytes, or $\geq 80 \% \mathbf{M}_{1}+\mathbf{M}_{2}$ monocytes were compared for their chemotactic activity. Enriched $M_{1}+M_{2}$ and $M_{3}$ monocytes were prepared by the thrombin aggregation method.

\$ Mean $\pm 1 \mathrm{SD}$ of cells migrating specifically toward chemoattractant for triplicate plates.

computer analysis of volume distributions suggested the presence of a third, intermediate-sized population, we termed these subpopulations $M_{1}, M_{2}$, and $M_{3}$ in order of increasing size. All three subpopulations were present in 30 normal individuals. In chemotaxis tests, large $\mathrm{M}_{3}$ monocytes were 10 times more responsive than the smaller $\mathrm{M}_{1}+\mathrm{M}_{2}$ monocytes.

Considerable evidence supports the contention that these volumetrically defined subpopulations of adherent cells are in fact subpopulations of monocytes. Adherent cells containing these three subpopulations were $89 \pm 5 \%$ monocytes by nonspecific esterase staining and phagocytosis of yeast and staphylococci. Because the percentage of cells in the $M_{1}, M_{2}$, and $M_{3}$ peaks always exceeded the percentage of nonmonocytes, it was clear that most of the cells in each subpopulation were monocytes. Additional evidence supporting the identity of these cells as monocytes included the ability of carbonyl iron particles to eliminate 95\% of the adherent cells under optimal conditions and the differentiation of enriched $\mathbf{M}_{1}+\mathbf{M}_{2}$ cells into macrophages in cell culture (unpublished data).

Although it was clear that adherent $\mathrm{M}_{1}, \mathrm{M}_{2}$, and $\mathrm{M}_{3}$ cells were indeed monocytes, it was conceivable that volumetric differences could be an artifactual effect of isolation and purification procedures rather than a true characteristic of monocyte subpopulations. However, extensive volume analysis of buffy-coat leukocytes, MNL, and cells obtained at each step of the purification process indicated that $\mathbf{M}_{1}, \dot{M}_{2}$, and $\mathbf{M}_{3}$ monocytes were normally present in blood and that purification procedures neither created nor appreciably altered these volume differences (data not shown). These findings strongly supported the existence of volumetrically definable monocyte subpopulations.

Having identified volumetric subpopulations of monocytes, we sought functional differences among them. Chemotaxis assays comparing iron-depleted monocytes to total monocytes demonstrated that $\mathrm{M}_{1}+\mathrm{M}_{2}$ monocytes were less active than total monocytes. Similar experiments using thrombin-purified fractions confirmed these results and localized essentially all of the chemotactic activity in the $\mathrm{M}_{3}$ subpopulation. In addition, preliminary experiments that used an adaptation of the agarose plate method in which migrating monocytes could be harvested and analyzed volumetrically indicated that the chemotactically active cells among whole $\mathrm{MNL}$ were $>90 \% \mathrm{M}_{3}$ monocytes (data not shown). These experiments provided clear evidence that volumetrically-defined monocyte subpopulations have markedly different functional capabilities. Although the effect of cell size on chemotaxis is unknown, it is unlikely that the 10-fold difference between $\mathrm{M}_{1}+\mathrm{M}_{2}$ and $\mathrm{M}_{3}$ monocytes can be attributed to size difference because there is less than a threefold difference in cell volume and less than a twofold difference in surface area.

Others have recently observed volumetric subpopulations of human monocytes and murine mononuclear phagocytes. Norris et al. (10) fractionated human MNL by elutriation and found a population of small peroxidase-positive and phagocytic cells in predominantly lymphocyte fractions. These cells were found to be relatively deficient in antibody-dependent cellular cytotoxicity compared to purified large monocytes. Breard et al. (11) identified two populations of human monocytes with a monocyte specific heteroantiserum that differed in size. Lee and Berry (12) used velocity sedimentation to fractionate Corynebacterium parvuminduced mouse peritoneal mononuclear phagocytes into small, medium, and large populations. Small and medium cells functioned as accessory cells during in vitro generation of antibody to sheep erythrocytes but were not cytotoxic for tumor cells. Conversely, the large cells were cytotoxic and either suppressed or were unable to serve as accessory cells in the antibody response.

The demonstration of volumetrically distinct and functionally different populations of human monocytes is consistent with the hypothesis that the mononuclear phagocyte system is composed of identifiable subsets with discrete functions. However, neither our data nor that of others elucidates whether these functionally diverse subpopulations represent cells following independent pathways of differentiation analogous to $T$ and B lymphocytes, or whether they represent transient but functionally discrete steps in a continuous dif- 
ferentiation sequence. The ontogeny and additional functional characteristics of monocyte subpopulations must now be determined.

In summary, we have demonstrated that cell volume differences provide a means of identifying and quantitating subpopulations of human blood monocytes that are functionally distinct. Additional studies of these subpopulations may substantially enhance our understanding of the mononuclear phagocyte system and its complex role in the host defenses of man.

\section{ACKNOWLEDGMENTS}

We thank Ms. Sylvia A. Rayner, Mr. Calvin Myers, Ms. Dorothy Haskett, and Ms. Sherrie Gard for excellent technical assistance. We also thank Dr. Robert I. Lehrer for his critical review of this manuscript.

This work was supported in part by grants CA12800 and CA22794 awarded by the National Cancer Institute, Department of Health, Education, and Welfare and contract EY76-S003-0034 awarded by the U. S. Department of Energy to the University of California.

\section{REFERENCES}

1. Walker, W. S. 1976. Functional heterogeneity of macrophages. In Immunobiology of the Macrophage. D. S. Nelson, editor. Academic Press, Inc., New York. 91-110.

2. Boyum, A. 1968. Isolation of leukocytes from human blood. Scand. J. Clin. Lab. Invest. 21(Suppl. 97): 51-76.

3. Tucker, S. B., R. V. Pierre, and R. E. Jordan. 1977. Rapid identification of monocytes in a mixed mononuclear cell preparation. J. Immunol. Methods. 14: 267-269.
4. Steincamp, J. A., M. J. Fulwyler, J. R. Coulter, R. D. Hiebert, J. L. Horney, and P. F. Mullaney. 1973. A new multiparameter separator for microscopic particles and biological cells. Rev. Sci. Instrum. 44: 1301-1310.

5. Rotman, B., and B. Papermaster. 1966. Membrane properties of living mammalian cells as studied by enzymatic hydrolysis of fluorogenic esters. Proc. Natl. Acad. Sci. U. S. A. 55: 134-141.

6. Holm, G., D. Pettersson, H. Mellstedt, E. Hedfors, and B. Bloth. 1975. Lymphocyte subpopulations in peripheral blood of healthy persons. Clin. Exp. Immunol. 20: 443-457.

7. Terasaki, P. I., D. Bernoco, M. S. Park, G. Ozturk, and Y. Iwaki. 1978. Microdroplet Testing for HLA-A-B-C and D Antigens. Am. J. Clin. Pathol. 69: 103-120.

8. Klein, R. B., T. J. Fischer, S. E. Gard, M. Biberstein, K. C. Rich, and E. R. Stiehm. 1977. Decreased mononuclear and polymorphonuclear chemotaxis in human newborns. Pediatrics 60: 467-472.

9. Cox, J. M., and T. P. Stossel. 1976. Measurement of phagocytosis by macrophages. In In Vitro Methods in Cell-Mediated and Tumor Immunity. B. R. Bloom and J. R. David, editors. Academic Press, Inc., New York. 363-368.

10. Norris, D. A., R. M. Morris, R. J. Sanderson, and P. F. Kohler, 1979. Isolation of functional subsets of human peripheral blood monocytes. J. Immunol. 123: 166-172.

11. Breard, J., H. Lazarus, and S. F. Schlossman. 1979. Generation and specificity of an heteroantiserum to human peripheral blood monocytes. Fed. Proc. 38: 1418. (Abstr.)

12. Lee, K. C., and D. Berry. 1977. Functional heterogeneity in macrophages activated by Corynebacterium parvum: characterization of subpopulations with different activities in promoting immune responses and suppressing tumor cell growth. J. Immunol. 118: 1530-1540. 